\title{
THE CONVERGENCE THEOREMS FOR COMMON FIXED POINTS OF UNIFORMLY L-LIPSCHITZIAN ASYMPTOTICALLY $\Phi$-PSEUDOCONTRACTIVE MAPPINGS
}

\author{
Zhiqun Xue
}

\begin{abstract}
In this paper, we show that the modified Mann iteration with errors converges strongly to fixed point for uniformly L-Lipschitzian asymptotically $\Phi$-pseudocontractive mappings in real Banach spaces.

Meanwhile, it is proved that the convergence of Mann and Ishikawa iterations is equivalent for uniformly L-Lipschitzian asymptotically $\Phi$ pseudocontractive mappings in real Banach spaces. Finally, we obtain the convergence theorems of Ishikawa iterative sequence and the modified Ishikawa iterative process with errors.
\end{abstract}

\section{Introduction}

In this paper, we assume that $E$ is a real Banach space, $E^{*}$ is the dual space of $E, D$ is a nonempty closed convex subset of $E$ and $J: E \rightarrow 2^{E^{*}}$ is the normalized duality mapping defined by

$$
J(x)=\left\{f \in E^{*}:\langle x, f\rangle=\|x\|^{2}=\|f\|^{2}\right\}, \quad \forall x \in E,
$$

where $\langle\cdot, \cdot\rangle$ denotes the generalized duality pairing. The single-valued normalized duality mapping is denoted by $j$.

Definition 1.1. Let $T: D \rightarrow D$ be a mapping.

(1) $T$ is called uniformly L-Lipschitzian if there is a constant $L>0$ such that for any $x, y \in D$,

$$
\left\|T^{n} x-T^{n} y\right\| \leq L\|x-y\|, \quad \forall n \geq 1 .
$$

(2) $T$ is called asymptotically pseudocontractive with a sequence $\left\{k_{n}\right\} \subset$ $[1,+\infty)$ and $\lim _{n \rightarrow \infty} k_{n}=1$ if for each $x, y \in D$, there exists $j(x-y) \in J(x-y)$ such that

$$
\left\langle T^{n} x-T^{n} y, j(x-y)\right\rangle \leq k_{n}\|x-y\|^{2}, \quad \forall n \geq 1 .
$$

Received October 16, 2008.

2000 Mathematics Subject Classification. 47H10 47H09 46 B20.

Key words and phrases. uniformly L-Lipschitzian, asymptotically $\Phi$-pseudocontractive mapping, modified Mann iterative process, modified Ishikawa iterative process, fixed point.

The Project is supported by Hebei province science and technology foundation(No. 072056197D) and the National Natural Science Foundation of China Grant 10872136. 
(3) $T$ is called asymptotically $\Phi$-pseudocontractive with a sequence $\left\{k_{n}\right\} \subset$ $[1,+\infty)$ and $\lim _{n \rightarrow \infty} k_{n}=1$ if for all $x, y \in D$, there exist $j(x-y) \in J(x-y)$ and a strictly increasing continuous function $\Phi:[0,+\infty) \rightarrow[0,+\infty)$ with $\Phi(0)=0$ such that

$$
\left\langle T^{n} x-T^{n} y, j(x-y)\right\rangle \leq k_{n}\|x-y\|^{2}-\Phi(\|x-y\|), \quad \forall n \geq 1 .
$$

It is obvious that if $T$ is an asymptotically $\Phi$-pseudocontractive mapping, then $T$ is an asymptotically pseudocontractive mapping. Conversely, it is not true. The convergence of Mann-type and Ishikawa-type iteration processes for uniformly L-Lipschitzian and asymptotically $\Phi$-pseudocontractive mappings in Banach spaces have been studied extensively by many authors, see for example $[1,2,3,6]$.

Recently, Ofoedu [3] gave iterative approximation problem of fixed points for uniformly L-Lipschitzian asymptotically pseudocontractive mappings in Banach spaces. The results are as following.

Theorem 1.2 ([3, Theorem 3.1]). Let $E$ be a real Banach space. Let $K$ be a nonempty closed and convex subset of $E, T: K \rightarrow K$ a uniformly $L$ Lipschitzian asymptotically pseudocontractive mapping with sequence $\left\{k_{n}\right\}_{n \geq 0}$ $\subset[1,+\infty), \lim _{n \rightarrow \infty} k_{n}=1$ such that $x^{*} \in F(T)=\{x \in K: T x=x\}$. Let $\left\{\alpha_{n}\right\}_{n \geq 0} \subset[0,1]$ be such that $\sum_{n \geq 0} \alpha_{n}=\infty, \sum_{n \geq 0} \alpha_{n}^{2}<\infty$ and $\sum_{n \geq 0} \alpha_{n}\left(k_{n}-\right.$ $1)<\infty$. For arbitrary $x_{0} \in K$ let $\left\{x_{n}\right\}_{n \geq 0}$ be iteratively defined by

$$
x_{n+1}=\left(1-\alpha_{n}\right) x_{n}+\alpha_{n} T^{n} x_{n}, \quad n \geq 0 .
$$

Suppose there exists a strictly increasing function $\Phi:[0,+\infty) \rightarrow[0,+\infty), \Phi(0)$ $=0$ such that

$$
\left\langle T^{n} x-x^{*}, j\left(x-x^{*}\right)\right\rangle \leq k_{n}\left\|x-x^{*}\right\|^{2}-\Phi\left(\left\|x-x^{*}\right\|\right), \quad \forall x \in K .
$$

Then, $\left\{x_{n}\right\}_{n \geq 0}$ is bounded.

Theorem 1.3 ([3, Theorem 3.2]). Let $E$ be a real Banach space. Let $K$ be a nonempty closed and convex subset of $E, T: K \rightarrow K$ a uniformly L-Lipschitz asymptotically pseudocontractive mapping with sequence $\left\{k_{n}\right\}_{n \geq 0} \subset[1,+\infty)$, $\lim _{n \rightarrow \infty} k_{n}=1$. Let $x^{*} \in F(T)=\{x \in K: T x=x\}$. Let $\left\{\alpha_{n}\right\}_{n>0} \subset[0,1]$ be such that $\sum_{n \geq 0} \alpha_{n}=\infty, \sum_{n \geq 0} \alpha_{n}^{2}<\infty$ and $\sum_{n \geq 0} \alpha_{n}\left(k_{n}-1\right)<\infty$. For arbitrary $x_{0} \in K$ let $\left\{x_{n}\right\}_{n \geq 0}$ be iteratively defined by

$$
x_{n+1}=\left(1-\alpha_{n}\right) x_{n}+\alpha_{n} T^{n} x_{n}, \quad n \geq 0 .
$$

Suppose there exists a strictly increasing continuous function $\Phi:[0,+\infty) \rightarrow$ $[0,+\infty), \Phi(0)=0$ such that

$$
\left\langle T^{n} x-x^{*}, j\left(x-x^{*}\right)\right\rangle \leq k_{n}\left\|x-x^{*}\right\|^{2}-\Phi\left(\left\|x-x^{*}\right\|\right), \quad \forall x \in K .
$$

Then, $\left\{x_{n}\right\}_{n \geq 0}$ converges strongly to $x^{*} \in F(T)$. 
Remark 1.4. As mentioned above Theorem 1.2 and Theorem 1.3, it is possible that $\Phi^{-1}\left(a_{0}\right)$ may be not sense while $\lim _{r \rightarrow+\infty} \Phi(r)=A<a_{0}$. For example, define $\Phi:[0,+\infty) \rightarrow[0,+\infty)$ by $\Phi(r)=\frac{r}{r+1}$, then $\Phi^{-1}(2)$ is senseless. Therefore, the proof of Theorem 3.1 and Theorem 3.2 in [3] is not reasonable. In order to avoid this problem, we will provide a significant improvement. Meanwhile, we obtain the convergence results of Ishikawa iterative sequence by the equivalence of the strong convergence results for uniformly L-Lipschitzian mappings in real Banach spaces. For this, we need the following concepts and lemmas.

Definition 1.5. Let $T_{1}, T_{2}: D \rightarrow D$ be two mappings.

(i) For any given $x_{1} \in D$, define the sequence $\left\{x_{n}\right\}_{n=1}^{\infty} \subset D$ by the iterative schemes

$$
\left\{\begin{array}{c}
y_{n}=\left(1-b_{n}-d_{n}\right) x_{n}+b_{n} T_{2}^{n} x_{n}+d_{n} w_{n}, \quad n \geq 1 \\
x_{n+1}=\left(1-a_{n}-c_{n}\right) x_{n}+a_{n} T_{1}^{n} y_{n}+c_{n} z_{n}, \quad n \geq 1
\end{array}\right.
$$

which is called the modified Ishikawa iterative process with errors, where $\left\{a_{n}\right\}_{n=1}^{\infty},\left\{b_{n}\right\}_{n=1}^{\infty},\left\{c_{n}\right\}_{n=1}^{\infty},\left\{d_{n}\right\}_{n=1}^{\infty}$ are real sequences in [0,1] satisfying conditions $a_{n}+c_{n} \leq 1, b_{n}+d_{n} \leq 1$, and $\left\{z_{n}\right\},\left\{w_{n}\right\}$ are the bounded sequences in $D$. If $b_{n}=d_{n}=0$, then the modified Ishikawa iterative process with errors reduces to:

(ii) For any given $u_{1} \in D$, define the sequence $\left\{u_{n}\right\}_{n=1}^{\infty} \subset D$ by the iterative schemes

$$
u_{n+1}=\left(1-a_{n}-c_{n}\right) u_{n}+a_{n} T_{1}^{n} u_{n}+c_{n} v_{n}, \quad n \geq 1,
$$

which is called the modified Mann iterative process with errors, where $\left\{v_{n}\right\}$ is the bounded sequence in $D$.

If $c_{n}=d_{n}=0$ in (1.1) and (1.2), then the corresponding iterations are called the modified Ishikawa and Mann iterations respectively, that is,

$$
\begin{gathered}
\left\{\begin{array}{c}
y_{n}=\left(1-b_{n}\right) x_{n}+b_{n} T_{2}^{n} x_{n}, \quad n \geq 1, \\
x_{n+1}=\left(1-a_{n}\right) x_{n}+a_{n} T_{1}^{n} y_{n}, \quad n \geq 1 ;
\end{array}\right. \\
u_{n+1}=\left(1-a_{n}\right) u_{n}+a_{n} T_{1}^{n} u_{n}, \quad n \geq 1 .
\end{gathered}
$$

Lemma 1.6 ([3]). Let $E$ be a real Banach space and let $J: E \rightarrow 2^{E^{*}}$ be a normalized duality mapping. Then

$$
\|x+y\|^{2} \leq\|x\|^{2}+2\langle y, j(x+y)\rangle
$$

for all $x, y \in E$ and each $j(x+y) \in J(x+y)$.

Lemma $1.7([4])$. Let $\left\{\rho_{n}\right\}_{n=1}^{\infty}$ be a nonnegative real numbers sequence satisfying the inequality

$$
\rho_{n+1} \leq\left(1-\theta_{n}\right) \rho_{n}+o\left(\theta_{n}\right),
$$

where $\theta_{n} \in(0,1)$ with $\sum_{n=1}^{\infty} \theta_{n}=\infty$. Then $\rho_{n} \rightarrow 0$ as $n \rightarrow \infty$. 


\section{Main results}

Theorem 2.1. Let $E$ be a real Banach space, $D$ be a nonempty closed convex subset of $E$ and $T_{1}: D \rightarrow D$ be a uniformly $L_{1}$-Lipschitzian asymptotically $\Phi$ pseudocontractive mapping with sequence $\left\{k_{1 n}\right\}_{n \geq 1} \subset[1,+\infty), \lim _{n \rightarrow \infty} k_{1 n}=$ 1. Let $q \in F\left(T_{1}\right)=\left\{x \in D: T_{1} x=x\right\}$. Let $\left\{a_{n}\right\}_{n \geq 1},\left\{c_{n}\right\}_{n \geq 1} \subset[0,1]$ be such that $\sum_{n>1} \alpha_{n}=\infty, \lim _{n \rightarrow \infty} a_{n}=0$ and $c_{n}=o\left(a_{n}\right)$ with $a_{n}+c_{n} \leq 1$, for any $n \geq 1$. The sequence $\left\{u_{n}\right\}_{n=1}^{\infty}$ is defined by (1.2). Then, $\left\{u_{n}\right\}_{n \geq 1}$ converges strongly to $q$.

Proof. Applying Lemma 1.6 and (1.2), we have

$$
\begin{aligned}
& \left\|u_{n+1}-q\right\|^{2} \\
= & \left\|\left(1-a_{n}-c_{n}\right)\left(u_{n}-q\right)+a_{n}\left(T_{1}^{n} u_{n}-q\right)+c_{n}\left(v_{n}-q\right)\right\|^{2} \\
\leq & \left(1-a_{n}-c_{n}\right)^{2}\left\|u_{n}-q\right\|^{2}+2 a_{n}\left\langle T_{1}^{n} u_{n}-q, j\left(u_{n+1}-q\right)\right\rangle \\
& +2 c_{n}\left\langle v_{n}-q, j\left(u_{n+1}-q\right)\right\rangle \\
\leq & \left(1-a_{n}-c_{n}\right)^{2}\left\|u_{n}-q\right\|^{2}+2 a_{n}\left\langle T_{1}^{n} u_{n}-T_{1}^{n} u_{n+1}, j\left(u_{n+1}-q\right)\right\rangle \\
& +2 a_{n}\left\langle T_{1}^{n} u_{n+1}-T_{1}^{n} q, j\left(u_{n+1}-q\right)\right\rangle+2 c_{n}\left\|v_{n}-q\right\| \cdot\left\|u_{n+1}-q\right\| \\
\leq & \left(1-a_{n}-c_{n}\right)^{2}\left\|u_{n}-q\right\|^{2}+2 a_{n} L_{1}\left\|u_{n}-u_{n+1}\right\| \cdot\left\|u_{n+1}-q\right\| \\
& +2 a_{n}\left(k_{1 n}\left\|u_{n+1}-q\right\|^{2}-\Phi\left(\left\|u_{n+1}-q\right\|\right)\right) \\
& +c_{n}\left\|v_{n}-q\right\|+c_{n}\left\|v_{n}-q\right\| \cdot\left\|u_{n+1}-q\right\|^{2} .
\end{aligned}
$$

From (1.2), we observe that

$$
\begin{aligned}
& \left\|u_{n}-u_{n+1}\right\| \\
\leq & a_{n}\left\|u_{n}-T_{1}^{n} u_{n}\right\|+c_{n}\left\|u_{n}-v_{n}\right\| \\
\leq & \left(a_{n}\left(1+L_{1}\right)+c_{n}\right)\left\|u_{n}-q\right\|+c_{n}\left\|v_{n}-q\right\| .
\end{aligned}
$$

Taking (2.2) into (2.1), we obtain

$$
\begin{aligned}
& \left\|u_{n+1}-q\right\|^{2} \\
\leq & \left(1-a_{n}-c_{n}\right)^{2}\left\|u_{n}-q\right\|^{2}+2 a_{n} L_{1}\left[\left(a_{n}\left(1+L_{1}\right)+c_{n}\right)\left\|u_{n}-q\right\|\right. \\
& \left.+c_{n}\left\|v_{n}-q\right\|\right] \cdot\left\|u_{n+1}-q\right\| \\
& +2 a_{n} k_{1 n}\left\|u_{n+1}-q\right\|^{2}-2 a_{n} \Phi\left(\left\|u_{n+1}-q\right\|\right)+c_{n}\left\|v_{n}-q\right\| \\
& +c_{n}\left\|v_{n}-q\right\| \cdot\left\|u_{n+1}-q\right\|^{2} \\
\leq & \left(1-a_{n}\right)^{2}\left\|u_{n}-q\right\|^{2} \\
& +a_{n} L_{1}\left[a_{n}\left(1+L_{1}\right)+c_{n}\right]\left(\left\|u_{n}-q\right\|^{2}+\left\|u_{n+1}-q\right\|^{2}\right) \\
& +a_{n} L_{1} c_{n}\left\|v_{n}-q\right\|\left(1+\left\|u_{n+1}-q\right\|^{2}\right)+2 a_{n} k_{1 n}\left\|u_{n+1}-q\right\|^{2} \\
& -2 a_{n} \Phi\left(\left\|u_{n+1}-q\right\|\right) \\
& +c_{n}\left\|v_{n}-q\right\|+c_{n}\left\|v_{n}-q\right\| \cdot\left\|u_{n+1}-q\right\|^{2} .
\end{aligned}
$$


It then follows from (2.3) that

(2.4)

$$
\begin{aligned}
& \leq u_{n+1}-q \|^{2} \\
& \leq \frac{\left(1-a_{n}\right)^{2}+a_{n} L_{1}\left[a_{n}\left(1+L_{1}\right)+c_{n}\right]}{1-2 a_{n} k_{1 n}-a_{n} L_{1}\left[a_{n}\left(1+L_{1}\right)+c_{n}\right]-\left(a_{n} L_{1}+1\right) c_{n}\left\|v_{n}-q\right\|}\left\|u_{n}-q\right\|^{2} \\
& \quad-\frac{2 a_{n}}{1-2 a_{n} k_{1 n}-a_{n} L_{1}\left[a_{n}\left(1+L_{1}\right)+c_{n}\right]-\left(a_{n} L_{1}+1\right) c_{n}\left\|v_{n}-q\right\|} \Phi\left(\left\|u_{n+1}-q\right\|\right) \\
& \quad+\frac{\left(a_{n} L_{1}+1\right) c_{n}\left\|v_{n}-q\right\|}{1-2 a_{n} k_{1 n}-a_{n} L_{1}\left[a_{n}\left(1+L_{1}\right)+c_{n}\right]-\left(a_{n} L_{1}+1\right) c_{n}\left\|v_{n}-q\right\|} .
\end{aligned}
$$

Since $2 a_{n} k_{1 n}+a_{n} L_{1}\left[a_{n}\left(1+L_{1}\right)+c_{n}\right]+\left(a_{n} L_{1}+1\right) c_{n}\left\|v_{n}-q\right\| \rightarrow 0$ as $n \rightarrow \infty$, then there exists $N$ such that $2 a_{n} k_{1 n}+a_{n} L_{1}\left[a_{n}\left(1+L_{1}\right)+c_{n}\right]+\left(a_{n} L_{1}+1\right) c_{n}\left\|v_{n}-q\right\|<$ $\frac{1}{2}, \forall n>N$, i.e., $1>1-2 a_{n} k_{1 n}-a_{n} L_{1}\left[a_{n}\left(1+L_{1}\right)+c_{n}\right]-\left(a_{n} L_{1}+1\right) c_{n}\left\|v_{n}-q\right\|>$ $\frac{1}{2}(n>N)$. Thus, we have

$$
\begin{aligned}
& \quad\left\|u_{n+1}-q\right\|^{2} \\
& \leq\left\|u_{n}-q\right\|^{2} \\
& \quad+a_{n} \frac{a_{n}+2\left(k_{1 n}-1\right)+2 L_{1}\left[a_{n}\left(1+L_{1}\right)+c_{n}\right]+\left(a_{n} L_{1}+1\right) c_{n} / a_{n}\left\|v_{n}-q\right\|}{1-2 a_{n} k_{1 n}-a_{n} L_{1}\left[a_{n}\left(1+L_{1}\right)+c_{n}\right]-\left(a_{n} L_{1}+1\right) c_{n}\left\|v_{n}-q\right\|}\left\|u_{n}-q\right\|^{2} \\
& \quad-\frac{2 a_{n}}{1-2 a_{n} k_{1 n}-a_{n} L_{1}\left[a_{n}\left(1+L_{1}\right)+c_{n}\right]-\left(a_{n} L_{1}+1\right) c_{n}\left\|v_{n}-q\right\|} \Phi\left(\left\|u_{n+1}-q\right\|\right) \\
& \\
& \quad+\frac{\left(a_{n} L_{1}+1\right) c_{n}\left\|v_{n}-q\right\|}{1-2 a_{n} k_{1 n}-a_{n} L_{1}\left[a_{n}\left(1+L_{1}\right)+c_{n}\right]-\left(a_{n} L_{1}+1\right) c_{n}\left\|v_{n}-q\right\|} \\
& \leq\left\|u_{n}-q\right\|^{2}+2 a_{n} A_{n}\left\|u_{n}-q\right\|^{2}-2 a_{n} \Phi\left(\left\|u_{n+1}-q\right\|\right)+B_{n},
\end{aligned}
$$

where $A_{n}=a_{n}+2\left(k_{1 n}-1\right)+2 L_{1}\left[a_{n}\left(1+L_{1}\right)+c_{n}\right]+\left(a_{n} L_{1}+1\right) c_{n} / a_{n}\left\|v_{n}-q\right\| \rightarrow 0$ as $n \rightarrow \infty, B_{n}=2\left(a_{n} L_{1}+1\right) c_{n}\left\|v_{n}-q\right\|=o\left(a_{n}\right)$.

Let $\inf _{n \geq N} \frac{\Phi\left(\left\|u_{n+1}-q\right\|\right)}{1+\left\|u_{n+1}-q\right\|^{2}}=\lambda$. Then $\lambda=0$. Suppose this is not the case, i.e., suppose $\lambda>0$, and choose a $\gamma>0$ such that $\gamma<\min \{1, \lambda\}$. Then $\frac{\Phi\left(\left\|u_{n+1}-q\right\|\right)}{1+\left\|u_{n+1}-q\right\|^{2}} \geq \gamma$, i.e., $\Phi\left(\left\|u_{n+1}-q\right\|\right) \geq \gamma+\gamma\left\|u_{n+1}-q\right\|^{2} \geq \gamma\left\|u_{n+1}-q\right\|^{2}$. And it results that

$$
\begin{aligned}
& \left\|u_{n+1}-q\right\|^{2} \\
\leq & \frac{1+2 a_{n} A_{n}}{1+2 a_{n} \gamma}\left\|u_{n}-q\right\|^{2}+\frac{B_{n}}{1+2 a_{n} \gamma} \\
= & \left(1-a_{n} \frac{2 \gamma-2 A_{n}}{1+2 a_{n} \gamma}\right)\left\|u_{n}-q\right\|^{2}+\frac{B_{n}}{1+2 a_{n} \gamma} .
\end{aligned}
$$

Since $a_{n}, A_{n} \rightarrow 0$ as $n \rightarrow \infty$, there exists $N_{1}>N$ such that $\frac{2 \gamma-2 A_{n}}{1+2 a_{n} \gamma}>\gamma$ for all $n>N_{1}$. Hence, from (2.6)

$$
\left\|u_{n+1}-q\right\|^{2} \leq\left(1-a_{n} \gamma\right)\left\|u_{n}-q\right\|^{2}+\frac{B_{n}}{1+2 a_{n} \gamma}
$$


for all $n>N_{1}$. Applying Lemma 1.7, we obtain that $\left\|u_{n+1}-q\right\| \rightarrow 0$ as $n \rightarrow \infty$. By the continuity of $\Phi$, then $\frac{\Phi\left(\left\|u_{n+1}-q\right\|\right)}{1+\left\|u_{n+1}-q\right\|^{2}} \rightarrow 0$ as $n \rightarrow \infty$. This is a contradiction and so $\lambda=0$. Consequently, there exists an infinite subsequence such that $\left\|u_{n_{j}+1}-q\right\| \rightarrow 0$ as $j \rightarrow \infty$. Next we want to prove that $\| u_{n_{j}+m}-$ $q \| \rightarrow 0$ as $j \rightarrow \infty$ by induction. Let $\forall \varepsilon \in(0,1)$, choose $n_{j}>N$ such that $\left\|u_{n_{j}+1}-q\right\|<\epsilon, A_{n_{j}+1}<\frac{\Phi(\epsilon)}{2\left(1+\epsilon^{2}\right)}, \frac{B_{n_{j}+1}}{2 a_{n_{j}+1}}<\frac{\Phi(\epsilon)}{4}$. First, we want to prove $\left\|u_{n_{j}+2}-q\right\|<\epsilon$. Suppose it is not this case. Then $\left\|u_{n_{j}+2}-q\right\| \geq \epsilon$, this implies $\Phi\left(\left\|u_{n_{j}+2}-q\right\|\right) \geq \Phi(\epsilon)$. Using the formula (2.5), then we may obtain the following estimates

$$
\begin{aligned}
& \left\|u_{n_{j}+2}-q\right\|^{2} \\
\leq & \left\|u_{n_{j}+1}-q\right\|^{2}+2 a_{n_{j}+1} A_{n_{j}+1}\left\|u_{n_{j}+1}-q\right\|^{2} \\
& \quad-2 a_{n_{j}+1} \Phi\left(\left\|u_{n_{j}+2}-q\right\|\right)+2 a_{n_{j}+1} \frac{B_{n_{j}+1}}{2 a_{n_{j}+1}} \\
& <\epsilon^{2}-2 a_{n_{j}+1} \frac{\Phi(\epsilon)}{4}<\epsilon^{2}
\end{aligned}
$$

is a contradiction. Hence $\left\|u_{n_{j}+2}-q\right\|<\epsilon$. Assume that it holds for $m=k$. Then by the argument above, we easily prove that it holds for $m=k+1$. Hence for $\forall m>1$, we obtain $\left\|u_{n_{j}+m}-q\right\|<\epsilon$. This completes the proof.

Remark 2.2. Theorem 2.1 improves and extends Theorem 3.1 and Theorem 3.2 in Ofoedu [3] in the following sense:

1. Theorem 2.1 differs greatly from Theorem 3.1 and Theorem 3.2 of Ofoedu [3] in the proof method.

2. The conditions $\sum_{n \geq 0} \alpha_{n}^{2}<\infty$ and $\sum_{n \geq 0} \alpha_{n}\left(k_{n}-1\right)<\infty$ in [3, Theorem 3.1 and Theorem 3.2] are replaced by the more general condition $\lim _{n \rightarrow \infty} \alpha_{n}=$ 0 , the conclusion still holds.

3. The Mann iteration method in [3] is extended to the modified Mann iteration method with errors introduced by $\mathrm{Xu}$ [5]. Therefore, while $c_{n}=0$, for any $n \geq 1$ in Theorem 2.1, then the following result holds.

Corollary 2.3. Let $E$ be a real Banach space, $D$ be a nonempty closed convex subset of $E$ and $T_{1}: D \rightarrow D$ be a uniformly $L_{1}$-Lipschitzian asymptotically $\Phi$-pseudocontractive mapping with sequence $\left\{k_{1 n}\right\}_{n \geq 1} \subset[1,+\infty)$ and $\lim _{n \rightarrow \infty} k_{1 n}=1$. Let $q \in F\left(T_{1}\right)=\left\{x \in D: T_{1} x=x\right\}$. Let $\left\{a_{n}\right\}_{n>1} \subset[0,1]$ be such that $\sum_{n \geq 1} \alpha_{n}=\infty$ and $\lim _{n \rightarrow \infty} a_{n}=0$. The sequence $\left\{u_{n}\right\}_{n=1}^{\infty}$ is defined by (1.4). Then, $\left\{u_{n}\right\}_{n \geq 1}$ converges strongly to $q$.

Theorem 2.4. Let $E$ be a real Banach space, $D$ be a nonempty closed convex subset of $E$ and $T_{i}: D \rightarrow D(i=1,2)$ be two uniformly L-Lipschitzian asymptotically $\Phi$-pseudocontractive mappings with the sequences $\left\{k_{1 n}\right\},\left\{k_{2 n}\right\} \subset[1$, $+\infty)$ and $\lim _{n \rightarrow \infty} k_{1 n}=\lim _{n \rightarrow \infty} k_{2 n}=1$. Let $q \in F\left(T_{1}\right) \cap F\left(T_{2}\right) \neq \emptyset$. Let $\left\{a_{n}\right\}_{n=1}^{\infty}$ and $\left\{b_{n}\right\}_{n=1}^{\infty}$ be two sequences in $[0,1]$ satisfying the following conditions: (i) $a_{n}, b_{n} \rightarrow 0$ as $n \rightarrow \infty$; (ii) $\sum_{n=1}^{\infty} a_{n}=\infty$. The sequences 
$\left\{u_{n}\right\}_{n=1}^{\infty}$ and $\left\{x_{n}\right\}_{n=1}^{\infty}$ are defined by (1.3) and (1.4), respectively. Then the following two assertions are equivalent:

(i) The modified Mann iteration (1.4) converges strongly to the fixed point $q$ of $T_{1}$;

(ii) The modified Ishikawa iteration (1.3) converges strongly to the common fixed point $q$ of $T_{1} \cap T_{2}$.

Proof. If the Ishikawa iteration (1.3) converges to the fixed point $q$, then by putting $b_{n}=0$, we can get the convergence of the Mann iteration (1.4). Conversely, we only need to prove (i) $\Rightarrow$ (ii), i.e., $\left\|u_{n}-q\right\| \rightarrow 0$ as $n \rightarrow$ $\infty \Rightarrow\left\|x_{n}-q\right\| \rightarrow 0$ as $n \rightarrow \infty$. Denote $L=\max \left\{L_{1}, L_{2}\right\}$, where $L_{1}$ and $L_{2}$ satisfy the inequality: $\left\|T_{i}^{n} x-T_{i}^{n} y\right\| \leq L_{i}\|x-y\|$ for $\forall x, y \in D$, $i=1,2 ; k_{n}=\max \left\{k_{1 n}, k_{2 n}\right\}$, where $\left\{k_{1 n}\right\}$ and $\left\{k_{2 n}\right\}$ satisfy the inequality: $\left\langle T_{i}^{n} x-T_{i}^{n} y, j(x-y)\right\rangle \leq k_{i n}\|x-y\|^{2}-\Phi(\|x-y\|), \forall x, y \in D, \forall n \geq 1, i=1,2$.

Applying (1.3), (1.4) and Lemma 1.6, we have

$$
\begin{aligned}
& \left\|x_{n+1}-u_{n+1}\right\|^{2} \\
= & \left\|\left(1-a_{n}\right)\left(x_{n}-u_{n}\right)+a_{n}\left(T_{1}^{n} y_{n}-T_{1}^{n} u_{n}\right)\right\|^{2} \\
\leq & \left(1-a_{n}\right)^{2}\left\|x_{n}-u_{n}\right\|^{2}+2 a_{n}\left\langle T_{1}^{n} y_{n}-T_{1}^{n} u_{n}, j\left(x_{n+1}-u_{n+1}\right)\right\rangle \\
\leq & \left(1-a_{n}\right)^{2}\left\|x_{n}-u_{n}\right\|^{2}+2 a_{n}\left\langle T_{1}^{n} y_{n}-T_{1}^{n} x_{n+1}, j\left(x_{n+1}-u_{n+1}\right)\right\rangle \\
& +2 a_{n}\left\langle T_{1}^{n} x_{n+1}-T_{1}^{n} u_{n+1}, j\left(x_{n+1}-u_{n+1}\right)\right\rangle \\
& +2 a_{n}\left\langle T_{1}^{n} u_{n+1}-T_{1}^{n} u_{n}, j\left(x_{n+1}-u_{n+1}\right)\right\rangle \\
\leq & \left(1-a_{n}\right)^{2}\left\|x_{n}-u_{n}\right\|^{2}+2 a_{n} L\left\|y_{n}-x_{n+1}\right\| \cdot\left\|x_{n+1}-u_{n+1}\right\| \\
& +2 a_{n}\left(k_{n}\left\|x_{n+1}-u_{n+1}\right\|^{2}-\Phi\left(\left\|x_{n+1}-u_{n+1}\right\|\right)\right) \\
& +2 a_{n} L\left\|u_{n+1}-u_{n}\right\| \cdot\left\|x_{n+1}-u_{n+1}\right\| .
\end{aligned}
$$

Observe that

$$
\begin{aligned}
& \left\|y_{n}-x_{n+1}\right\| \\
= & \left\|a_{n}\left(x_{n}-T_{1}^{n} y_{n}\right)-b_{n}\left(x_{n}-T_{2}^{n} x_{n}\right)\right\| \\
\leq & \left(a_{n}+b_{n}+b_{n} L_{2}\right)\left\|x_{n}-q\right\|+a_{n} L_{1}\left\|y_{n}-q\right\| \\
\leq & \left(a_{n}+b_{n}+b_{n} L_{2}\right)\left\|x_{n}-q\right\|+a_{n} L_{1}\left(1+b_{n} L_{2}\right)\left\|x_{n}-q\right\| \\
\leq & \left(a_{n}+b_{n}+b_{n} L+a_{n} L+a_{n} b_{n} L^{2}\right)\left\|x_{n}-q\right\| \\
\leq & \gamma_{n}\left(\left\|x_{n}-u_{n}\right\|+\left\|u_{n}-q\right\|\right),
\end{aligned}
$$

where $\gamma_{n}=a_{n}+b_{n}+b_{n} L+a_{n} L+a_{n} b_{n} L^{2}$.

Substituting (2.9) into (2.8), we obtain

$$
\begin{aligned}
& \left\|x_{n+1}-u_{n+1}\right\|^{2} \\
\leq & \left(1-a_{n}\right)^{2}\left\|x_{n}-u_{n}\right\|^{2}+2 a_{n} L \gamma_{n}\left(\left\|x_{n}-u_{n}\right\|+\left\|u_{n}-q\right\|\right) \cdot\left\|x_{n+1}-u_{n+1}\right\|
\end{aligned}
$$




$$
\begin{aligned}
& +2 a_{n}\left(k_{n}\left\|x_{n+1}-u_{n+1}\right\|^{2}-\Phi\left(\left\|x_{n+1}-u_{n+1}\right\|\right)\right) \\
& +2 a_{n} L\left\|u_{n+1}-u_{n}\right\| \cdot\left\|x_{n+1}-u_{n+1}\right\| \\
\leq & \left(1-a_{n}\right)^{2}\left\|x_{n}-u_{n}\right\|^{2}+a_{n} L \gamma_{n}\left(\left\|x_{n}-u_{n}\right\|^{2}+\left\|u_{n}-q\right\|^{2}+2\left\|x_{n+1}-u_{n+1}\right\|^{2}\right) \\
& +2 a_{n}\left(k_{n}\left\|x_{n+1}-u_{n+1}\right\|^{2}-\Phi\left(\left\|x_{n+1}-u_{n+1}\right\|\right)\right) \\
& +a_{n} L\left\|u_{n+1}-u_{n}\right\|\left(1+\left\|x_{n+1}-u_{n+1}\right\|^{2}\right) .
\end{aligned}
$$

Without loss of generality, we assume that

$$
0<1-2 a_{n} k_{n}-2 a_{n} \gamma_{n} L-a_{n} L\left\|u_{n+1}-u_{n}\right\|<1
$$

for any $n \geq 1$. Then (2.10) implies that

$$
\begin{aligned}
& \left\|x_{n+1}-u_{n+1}\right\|^{2} \\
& \leq \frac{\left(1-a_{n}\right)^{2}+a_{n} \gamma_{n} L}{1-2 a_{n} k_{n}-2 a_{n} \gamma_{n} L-a_{n} L\left\|u_{n+1}-u_{n}\right\|}\left\|x_{n}-u_{n}\right\|^{2} \\
& \quad+\frac{a_{n} L \gamma_{n}\left\|u_{n}-q\right\|^{2}+a_{n} L\left\|u_{n+1}-u_{n}\right\|}{1-2 a_{n} k_{n}-2 a_{n} \gamma_{n} L-a_{n} L\left\|u_{n+1}-u_{n}\right\|} \\
& \quad-\frac{2 a_{n}}{1-2 a_{n} k_{n}-2 a_{n} \gamma_{n} L-a_{n} L\left\|u_{n+1}-u_{n}\right\|} \Phi\left(\left\|x_{n+1}-u_{n+1}\right\|\right) .
\end{aligned}
$$

Since $2 a_{n} k_{n}+2 a_{n} \gamma_{n} L+a_{n} L\left\|u_{n+1}-u_{n}\right\| \rightarrow 0$ as $n \rightarrow \infty$, then there exists $N$ such that $2 a_{n} k_{n}+2 a_{n} \gamma_{n} L+a_{n} L\left\|u_{n+1}-u_{n}\right\|<\frac{1}{2}, \forall n>N$, i.e., $1>$ $1-2 a_{n} k_{n}-2 a_{n} \gamma_{n} L-a_{n} L\left\|u_{n+1}-u_{n}\right\|>\frac{1}{2}(n>N)$. Thus, we have

$$
\begin{aligned}
& \left\|x_{n+1}-u_{n+1}\right\|^{2} \\
\leq & \left\|x_{n}-u_{n}\right\|^{2}+2 a_{n} \frac{a_{n}+\left(k_{n}-1\right)+\gamma_{n} L+L\left\|u_{n+1}-u_{n}\right\|}{1-2 a_{n} k_{n}-2 a_{n} \gamma_{n} L-a_{n} L\left\|u_{n+1}-u_{n}\right\|}\left\|x_{n}-u_{n}\right\|^{2} \\
& +2 a_{n} \frac{L \gamma_{n}\left\|u_{n}-q\right\|^{2}+L\left\|u_{n+1}-u_{n}\right\|}{1-2 a_{n} k_{n}-2 a_{n} \gamma_{n} L-a_{n} L\left\|u_{n+1}-u_{n}\right\|} \\
& -\frac{2 a_{n}}{1-2 a_{n} k_{n}-2 a_{n} \gamma_{n} L-a_{n} L\left\|u_{n+1}-u_{n}\right\|} \Phi\left(\left\|x_{n+1}-u_{n+1}\right\|\right) \\
\leq & \left\|x_{n}-u_{n}\right\|^{2}+\frac{2 a_{n} B_{n}}{1-2 a_{n} k_{n}-2 a_{n} \gamma_{n} L-a_{n} L\left\|u_{n+1}-u_{n}\right\|}\left\|x_{n}-u_{n}\right\|^{2} \\
& +\frac{2 a_{n} C_{n}}{1-2 a_{n} k_{n}-2 a_{n} \gamma_{n} L-a_{n} L\left\|u_{n+1}-u_{n}\right\|} \\
& -\frac{2 a_{n}}{1-2 a_{n} k_{n}-2 a_{n} \gamma_{n} L-a_{n} L\left\|u_{n+1}-u_{n}\right\|} \Phi\left(\left\|x_{n+1}-u_{n+1}\right\|\right) \\
\leq & \left\|x_{n}-u_{n}\right\|^{2}+4 a_{n} B_{n}\left\|x_{n}-u_{n}\right\|^{2}+4 a_{n} C_{n}-2 a_{n} \Phi\left(\left\|x_{n+1}-u_{n+1}\right\|\right),
\end{aligned}
$$

where $B_{n}=a_{n}+\left(k_{n}-1\right)+\gamma_{n} L+L\left\|u_{n+1}-u_{n}\right\|, C_{n}=L \gamma_{n}\left\|u_{n}-q\right\|^{2}+L \| u_{n+1}-$ $u_{n} \|$. 
Set $\inf _{n \geq N} \frac{\Phi\left(\left\|x_{n+1}-u_{n+1}\right\|\right)}{1+\left\|x_{n+1}-u_{n+1}\right\|^{2}}=\lambda$. Then $\lambda=0$. If it is not the case, assume that $\lambda>0$. Let $0<\gamma<\min \{1, \lambda\}$. Then $\frac{\Phi\left(\left\|x_{n+1}-u_{n+1}\right\|\right)}{1+\left\|x_{n+1}-u_{n+1}\right\|^{2}} \geq \gamma$, i.e.,

$$
\Phi\left(\left\|x_{n+1}-u_{n+1}\right\|\right) \geq \gamma+\gamma\left\|x_{n+1}-u_{n+1}\right\|^{2} \geq \gamma\left\|x_{n+1}-u_{n+1}\right\|^{2} .
$$

Thus

$$
\begin{aligned}
& \left\|x_{n+1}-u_{n+1}\right\|^{2} \\
\leq & \frac{1+4 a_{n} B_{n}}{1+2 a_{n} \gamma}\left\|x_{n}-u_{n}\right\|^{2}+\frac{4 a_{n} C_{n}}{1+2 a_{n} \gamma} \\
= & \left(1-a_{n} \frac{2 \gamma-4 B_{n}}{1+2 a_{n} \gamma}\right)\left\|x_{n}-u_{n}\right\|^{2}+\frac{4 a_{n} C_{n}}{1+2 a_{n} \gamma} .
\end{aligned}
$$

By $a_{n}, B_{n} \rightarrow 0$ as $n \rightarrow \infty$, we choose $N_{1}>N$ such that $\frac{2 \gamma-4 B_{n}}{1+2 \alpha_{n} \gamma}>\gamma$ for all $n>N_{1}$. It follows from (2.13) that

$$
\left\|x_{n+1}-u_{n+1}\right\|^{2} \leq\left(1-a_{n} \gamma\right)\left\|x_{n}-u_{n}\right\|^{2}+\frac{4 a_{n} C_{n}}{1+2 a_{n} \gamma}
$$

for all $n>N_{1}$. It follows from Lemma 1.7 that $\left\|x_{n+1}-u_{n+1}\right\| \rightarrow 0$ as $n \rightarrow \infty$, this is a contradiction and so $\lambda=0$. Consequently, there exists an infinite subsequence such that $\left\|x_{n_{j}+1}-u_{n_{j}+1}\right\| \rightarrow 0$ as $j \rightarrow \infty$. Next we want to prove that $\left\|x_{n_{j}+m}-u_{n_{j}+m}\right\| \rightarrow 0$ as $j \rightarrow \infty$ by induction. Let $\forall \varepsilon \in(0,1)$, choose $n_{j}>N$ such that $\left\|x_{n_{j}+1}-u_{n_{j}+1}\right\|<\epsilon, B_{n_{j}+1}<\frac{\Phi(\epsilon)}{8\left(1+\epsilon^{2}\right)}, C_{n_{j}+1}<\frac{\Phi(\epsilon)}{8}$. First we want to prove $\left\|x_{n_{j}+2}-u_{n_{j}+2}\right\|<\epsilon$. Suppose it is not this case. Then $\left\|x_{n_{j}+2}-u_{n_{j}+2}\right\| \geq \epsilon$, this implies $\Phi\left(\left\|x_{n_{j}+2}-u_{n_{j}+2}\right\|\right) \geq \Phi(\epsilon)$. Using the formula (2.12), we now obtain the following estimates:

$$
\begin{aligned}
& \left\|x_{n_{j}+2}-u_{n_{j}+2}\right\|^{2} \\
\leq & \left\|x_{n_{j}+1}-u_{n_{j}+1}\right\|^{2}+4 a_{n_{j}+1} B_{n_{j}+1}\left\|x_{n_{j}+1}-u_{n_{j}+1}\right\|^{2} \\
& +4 a_{n_{j}+1} C_{n_{j}+1}-2 a_{n_{j}+1} \Phi\left(\left\|x_{n_{j}+2}-u_{n_{j}+2}\right\|\right) \\
< & \epsilon^{2}-a_{n_{j}+1} \Phi(\epsilon) \leq \epsilon^{2}
\end{aligned}
$$

is a contradiction. Hence $\left\|x_{n_{j}+2}-u_{n_{j}+2}\right\|<\epsilon$. Assume that it holds for $m=k$. Then by the argument above, we easily prove that it holds for $m=k+1$. Hence, we obtain $\left\|x_{n}-u_{n}\right\| \rightarrow 0$ as $n \rightarrow \infty$. Since $\left\|u_{n}-q\right\| \rightarrow 0$ as $n \rightarrow \infty$. From the inequality $0 \leq\left\|x_{n}-q\right\| \leq\left\|x_{n}-u_{n}\right\|+\left\|u_{n}-q\right\|$, we get $\left\|x_{n}-q\right\| \rightarrow 0$ as $n \rightarrow \infty$.

Theorem 2.5. Let $E$ be a real Banach space, $D$ be a nonempty closed and convex subset of $E$ and $T_{i}: D \rightarrow D(i=1,2)$ be two uniformly L-Lipschitzian asymptotically $\Phi$-pseudocontractive mappings with the sequences $\left\{k_{1 n}\right\},\left\{k_{2 n}\right\} \subset$ $[1,+\infty)$ and $\lim _{n \rightarrow \infty} k_{1 n}=\lim _{n \rightarrow \infty} k_{2 n}=1$. Let $q \in F\left(T_{1}\right) \cap F\left(T_{2}\right) \neq \emptyset$. Let $\left\{a_{n}\right\}_{n=1}^{\infty}$ and $\left\{b_{n}\right\}_{n=1}^{\infty}$ be two sequences in $[0,1]$ satisfying the following conditions: (i) $a_{n}, b_{n} \rightarrow 0$ as $n \rightarrow \infty$; (ii) $\sum_{n=1}^{\infty} a_{n}=\infty$. Then the modified Ishikawa iteration sequence (1.3) $\left\{x_{n}\right\}_{n=1}^{\infty}$ converges strongly to the common fixed point $q$ of $T_{1} \cap T_{2}$. 
Proof. Using Corollary 2.3 and Theorem 2.4, we obtain the conclusion of Theorem 2.5 .

Similarly, we also obtain the following results.

Theorem 2.6. Let $E$ be a real Banach space, $D$ be a nonempty closed convex subset of $E$ and $T_{i}: D \rightarrow D(i=1,2)$ be two uniformly L-Lipschitzian asymptotically $\Phi$-pseudocontractive mappings with the sequences $\left\{k_{1 n}\right\},\left\{k_{2 n}\right\} \subset[1$, $+\infty)$ and $\lim _{n \rightarrow \infty} k_{1 n}=\lim _{n \rightarrow \infty} k_{2 n}=1$. Let $q \in F\left(T_{1}\right) \cap F\left(T_{2}\right) \neq \emptyset$. Let $\left\{a_{n}\right\}_{n=1}^{\infty},\left\{b_{n}\right\}_{n=1}^{\infty},\left\{c_{n}\right\}_{n=1}^{\infty}$ and $\left\{d_{n}\right\}_{n=1}^{\infty}$ be the sequences in $[0,1]$ satisfying the following conditions: (i) $a_{n}+c_{n} \leq 1, b_{n}+d_{n} \leq 1$ for any $n \geq 1$; (ii) $a_{n}, b_{n}, d_{n} \rightarrow 0$ as $n \rightarrow \infty$; (iii) $\sum_{n=1}^{\infty} a_{n}=\infty$; (iv) $c_{n}=o\left(a_{n}\right)$. And let $\left\{z_{n}\right\}$ and $\left\{w_{n}\right\}$ be the bounded sequences in D. Suppose that the sequences $\left\{u_{n}\right\}_{n=1}^{\infty}$ and $\left\{x_{n}\right\}_{n=1}^{\infty}$ are defined by (1.2) and (1.1) respectively. Then the following two assertions are equivalent:

(i) The modified Mann iteration with errors (1.2) converges strongly to the fixed point $q$ of $T_{1}$;

(ii) The modified Ishikawa iteration with errors (1.1) converges strongly to the common fixed point $q$ of $T_{1} \cap T_{2}$.

Theorem 2.7. Let $E$ be a real Banach space, $D$ be a nonempty closed convex subset of $E$ and $T_{i}: D \rightarrow D(i=1,2)$ be two uniformly L-Lipschitzian asymptotically $\Phi$-pseudocontractive mappings with the sequences $\left\{k_{1 n}\right\},\left\{k_{2 n}\right\} \subset[1$, $+\infty)$ and $\lim _{n \rightarrow \infty} k_{1 n}=\lim _{n \rightarrow \infty} k_{2 n}=1$. Let $q \in F\left(T_{1}\right) \cap F\left(T_{2}\right) \neq \emptyset$. Let $\left\{a_{n}\right\}_{n=1}^{\infty},\left\{b_{n}\right\}_{n=1}^{\infty},\left\{c_{n}\right\}_{n=1}^{\infty}$ and $\left\{d_{n}\right\}_{n=1}^{\infty}$ be the sequences in $[0,1]$ satisfying the following conditions: (i) $a_{n}+c_{n} \leq 1, b_{n}+d_{n} \leq 1$ for any $n$; (ii) $a_{n}, b_{n}, d_{n} \rightarrow 0$ as $n \rightarrow \infty$; (iii) $\sum_{n=1}^{\infty} a_{n}=\infty$; (iv) $c_{n}=o\left(a_{n}\right)$. And let $\left\{z_{n}\right\}$ and $\left\{w_{n}\right\}$ be the bounded sequences in D. Suppose that the sequence $\left\{x_{n}\right\}_{n=1}^{\infty}$ is defined by (1.1). Then the modified Ishikawa iteration with errors (1.1) converges strongly to the common fixed point $q$ of $T_{1} \cap T_{2}$.

Proof. Using Theorem 2.1 and Theorem 2.6, we get the results of Theorem 2.7 .

\section{References}

[1] S. S. Chang, Some results for asymptotically pseudo-contractive mappings and asymptotically nonexpansive mappings, Proc. Amer. Math. Soc. 129 (2001), no. 3, 845-853.

[2] Y. J. Cho, J. I. Kang, and H. Y. Zhou, Approximating common fixed points of asymptotically nonexpansive mappings, Bull. Korean Math. Soc. 42 (2005), no. 4, 661-670.

[3] E. U. Ofoedu, Strong convergence theorem for uniformly L-Lipschitzian asymptotically pseudocontractive mapping in real Banach space, J. Math. Anal. Appl. 321 (2006), no. 2, 722-728.

[4] X. Weng, Fixed point iteration for local strictly pseudo-contractive mapping, Proc. Amer. Math. Soc. 113 (1991), no. 3, 727-731.

[5] Y. Xu, Ishikawa and Mann iterative processes with errors for nonlinear strongly accretive operator equations, J. Math. Anal. Appl. 224 (1998), no. 1, 91-101. 
[6] L. C. Zeng, Iterative approximation of fixed points of asymptotically pseudo-contractive mappings in uniformly smooth Banach spaces, Chinese Ann. Math. Ser. A 26 (2005), no. $2,283-290$.

Department of Mathematics and Physics

SHiJiAZHUANG RAILWAY Institute

ShiJiazhuang 050043, P. R. China

E-mail address: xuezhiqun@126.com 